\title{
Sulindac plus a phospholipid is effective for polyp reduction and safer than sulindac alone in a mouse model of colorectal cancer development
}

Jennifer S. Davis ${ }^{1 *}$ (D) Preeti Kanikarla-Marie², Mihai Gagea ${ }^{3}$, Patrick L. Yu ${ }^{4}$, Dexing Fang ${ }^{4}$, Manu Sebastian ${ }^{5}$, Peiying Yang ${ }^{6}$, Ernest Hawk ${ }^{7}$, Roderick Dashwood ${ }^{8}$, Lenard M. Lichtenberger ${ }^{4}$, David Menter ${ }^{2}$ and Scott Kopetz ${ }^{2}$

\begin{abstract}
Background: Non-steroidal anti-inflammatory drugs (NSAIDs) such as aspirin and sulindac are effective for colorectal cancer prevention in humans and some animal models, but concerns over gastro-intestinal (GI) ulceration and bleeding limit their potential for chemopreventive use in broader populations. Recently, the combination of aspirin with a phospholipid, packaged as PL-ASA, was shown to reduce GI toxicity in a small clinical trial. However, these studies were done for relatively short periods of time. Since prolonged, regular use is needed for chemopreventive benefit, it is important to know whether Gl safety is maintained over longer use periods and whether cancer prevention efficacy is preserved when an NSAID is combined with a phospholipid.
\end{abstract}

Methods: As a first step to answering these questions, we treated seven to eight-week-old, male and female C57B/6 Apc $\mathrm{min} /+$ mice with the NSAID sulindac, with and without phosphatidylcholine (PC) for 3-weeks. At the end of the treatment period, we evaluated polyp burden, gastric toxicity, urinary prostaglandins (as a marker of sulindac target engagement), and blood chemistries.

Results: Both sulindac and sulindac-PC treatments resulted in significantly reduced polyp burden, and decreased urinary prostaglandins, but sulindac-PC treatment also resulted in the reduction of gastric lesions compared to sulindac alone.

Conclusions: Together these data provide pre-clinical support for combining NSAIDs with a phospholipid, such as phosphatidylcholine to reduce Gl toxicity while maintaining chemopreventive efficacy.

Keywords: Colorectal cancer, Chemoprevention, Gastrointestinal safety, Sulindac, Polyps

\footnotetext{
* Correspondence: JSDavis@MDAnderson.org

${ }^{1}$ Departments of Epidemiology, The University of Texas, MD Anderson

Cancer Center, PO Box 301439, Houston, TX 77230-1439, USA

Full list of author information is available at the end of the article
}

(c) The Author(s). 2020 Open Access This article is licensed under a Creative Commons Attribution 4.0 International License, which permits use, sharing, adaptation, distribution and reproduction in any medium or format, as long as you give appropriate credit to the original author(s) and the source, provide a link to the Creative Commons licence, and indicate if changes were made. The images or other third party material in this article are included in the article's Creative Commons licence, unless indicated otherwise in a credit line to the material. If material is not included in the article's Creative Commons licence and your intended use is not permitted by statutory regulation or exceeds the permitted use, you will need to obtain permission directly from the copyright holder. To view a copy of this licence, visit http://creativecommons.org/licenses/by/4.0/. The Creative Commons Public Domain Dedication waiver (http://creativecommons.org/publicdomain/zero/1.0/) applies to the data made available in this article, unless otherwise stated in a credit line to the data. 


\section{Background}

Aspirin and non-aspirin non-steroidal anti-inflammatory drugs (NSAIDs) are increasingly recognized as effective chemoprevention agents against colorectal cancer (CRC) $[1,2]$. However, the broader use of aspirin for CRC prevention is greatly limited due to the significant risk of gastro-intestinal (GI) ulceration and bleeding resulting from prolonged, regular use in humans [3]. In the cardiovascular disease (CVD) prevention realm, several strategies have emerged to reduce the risk of gastric ulceration, including enteric coatings [4] and co-administration with a proton pump inhibitor (PPI) [5]. In the case of enteric coatings, they do not always lower-GI injury [6] and may interfere with the anti-platelet effects of aspirin [7]. Although effective for GI injury prevention, the long-term safety of PPIs has recently come into question [8], limiting consumer options for GI protection from NSAID induced GI injury.

One potential mechanism for GI injury is disruption of the hydrophobic gastric surface mucosa by aspirin and non-aspirin NSAIDs, exposing the epithelium to gastric acid, leading to ulceration [9-11]. The addition of a phospholipid [10-12] to aspirin and non-aspirin NSAIDs may reduce disruption of the hydrophobic mucosa and holds promise as an emerging strategy to reduce gastric ulceration. Moreover, the combination of aspirin with the phospholipid phosphatidylcholine (PC) recently attained FDA approval following a successful clinical trial demonstrating bioequivalence to immediaterelease aspirin [13]. Importantly, a separate, successful clinical trial demonstrated significantly reduced gastric ulceration in participants receiving PL-ASA (aspirin plus PC) compared to those receiving immediate-release aspirin [14]. Although these results are promising, PL-ASA has not been commercially available for a sufficient time to demonstrate long-term safety with prolonged use, as is needed for CRC prevention benefit [2]. Further, although PL-ASA has equivalent anti-pyretic, anti-inflammatory and anti-platelet properties as traditional aspirin, its chemopreventive properties are still being evaluated in vivo [15].

Sharing genetic etiology with the human Familial Adenomatous Polyposis (FAP) syndrome, the $A p c^{\mathrm{min} /+}$ mouse harbors a heterozygous truncating mutation in the Apc gene, leading to the formation of many polyps throughout the intestinal tract. Unlike humans with FAP, $A p c^{\mathrm{min} /+}$ mice develop most of their lesions in the small intestine, with infrequent development of colon tumors. Also, in contrast to FAP patients, Apc$\mathrm{min} /+$ mice rarely progress to adenocarcinoma, instead becoming moribund due to intestinal polyp burden and resulting anemia. Despite these dissimilarities, this model has proven useful for testing many chemopreventive agents including non-aspirin NSAIDs, the selective cyclooxygenase-2 (COX-2) inhibitor celecoxib, curcumin, and fish oil [16-21].
While NSAIDs such as sulindac, ibuprofen and piroxicam have demonstrated consistent efficacy in this model, aspirin studies in $A p c^{\text {min/+ }}$ mice have yielded mixed results [22-27]. Based on consistent findings of chemopreventive benefit of sulindac for humans with FAP [28] and faithfulness of the mouse model to recapitulate this benefit [21], we conducted studies in $A p c^{\mathrm{min} /+}$ mice to test the chemopreventive efficacy and safety of sulindac pre-associated with PC. To our knowledge, this is the first report of sulindac combined with PC.

\section{Methods \\ Animals}

All procedures were reviewed and approved by MD Anderson's Institutional Animal Care and Use Committee. $A p c^{\mathrm{min} /+}$ mice on the $\mathrm{C} 57 \mathrm{~B} / 6$ background were previously obtained from JAX (stock 002020) and a local breeding colony was established in a specific pathogen free environment. Mice were group housed in individually ventilated cages with a HEPA filtered air supply and blower exhaust. All cages had corn cob bedding and a Nestlet $^{\ominus}$ for enrichment. To the extent possible, mice were group housed with 2-3 animals per experimental cage. In the rare instances where individual housing was necessary, due to fighting, mice were provided a paper hut in addition to the Nestlet ${ }^{\circ}$. Chlorinated, reverse osmosis water was provided ad libitum via a valve at the rear of the cage. Mice were provided with Purina PicoLab Rodent Diet (5053, Purina), ad libitum. At 7 to 8 weeks of age (mean $=8.0$, range: 7.4-8.4), male and female mice (mean weight $=20.7 \mathrm{~g}$, range: $15.9-25.4$ ) were randomized to receive one of three controls, or one of two treatments (Table 1). The control groups included no treatment (6 mice), PBS (7 mice), and PC (volume equivalent to $30 \mathrm{mg} / \mathrm{kg}$ sulindac, 7 mice). The treatment groups included sulindac $(30 \mathrm{mg} / \mathrm{kg}, 7 \mathrm{mice})$ and sulindac-PC (30 mg NSAID $/ \mathrm{kg}, 6$ mice). A sulindac dose of $30 \mathrm{mg} / \mathrm{kg}$ per day was chosen based on prior experience and approximates $150 \mathrm{mg} /$ day in an adult human [29]. Sample size was chosen based on a power calculation to detect a $40 \%$ decrease in intestinal polyp count between untreated and sulindac treated mice at the end of study. With a minimum sample size of 6 per group, we had $80 \%$ power to detect a $40 \%$ decrease at a $p$ value of 0.01 . Polyp burden, defined as total intestinal polyp area, was also assessed. Mice were administered PBS, $\mathrm{PC}$, sulindac or sulindac-PC by daily oral gavage, using a soft-tip flexible gavage needle (Instech, FTP1838) for 3 weeks. Treatment length of 3 weeks was chosen as the time needed to reduce intestinal polyp count by at least $40 \%$, which was the basis of our power calculation. Treatments were conducted in the morning in the animal's home cage. Daily oral gavage was chosen, as it more closely resembles the mechanism of exposure in 
Table 1 Baseline animal characteristics

\begin{tabular}{|c|c|c|c|c|c|}
\hline & No Treatment & PBS & PC & Sulindac & Sulindac-PC \\
\hline$n$ & 6 & 7 & 7 & 7 & 6 \\
\hline \multicolumn{6}{|l|}{ Sex n (\%) } \\
\hline male & $3(50)$ & $4(57)$ & $4(57)$ & $4(57)$ & $2(33)$ \\
\hline female & $3(50)$ & $3(43)$ & $3(43)$ & $3(43)$ & $4(67)$ \\
\hline Age, weeks (SD) & $8.2(0.2)$ & $8.0(0.2)$ & $8.1(0.2)$ & $7.8(0.1)$ & $7.9(0.2)$ \\
\hline Weight, grams (SD) & $20.4(2.2)$ & $20.5(3.5)$ & $21.7(3.2)$ & $21.0(3.2)$ & 19.9 (3.6) \\
\hline
\end{tabular}

humans. Mice randomized to no treatment were restrained daily to control for the stress of daily manual restraint. Randomization and study entrance were conducted on a rolling basis, as animals became available, aiming to balance sex and age within each treatment or control group. Sulindac (Sigma) from a single batch was either combined with PC ((Lipoid S 100) Lipoid GmbH, Germany) as previously described [30] or on its own was diluted in PBS to a working concentration of $5 \mathrm{mg} / \mathrm{mL}$ and sonicated in a sonicating water bath for $30 \mathrm{~min}$ at room temperature (Branson 1800). The individual structures of sulindac (CAS: 38194-50-2) and PC (CAS: 97281-47-5) are known and have been previously published [15, 31]. Fresh aliquots were prepared each day prior to administration. Mice were weighed twice weekly and monitored for overall health condition and dose levels were adjusted once per week based on weight. At the completion of the treatment course, mice were euthanized via carbon dioxide asphyxiation, followed by cervical dislocation. Blood and urine samples were collected, and necropsy was performed in all mice. During necropsy the stomach was examined and evaluated for the presence of ulcers, and the intestinal tract for presence of mucosal polyps. Briefly, the intestinal tract, from the duodenum to the rectum, was excised in-tact, flushed with PBS, expanded with freshly prepared 1.1\% paraformaldehyde, 1.25\%glutaraldehyde (in PBS) and fixed in this solution at $4{ }^{\circ} \mathrm{C}$ for $72 \mathrm{~h}$. Mouse treatment identification was blinded at necropsy, where each mouse was assigned a 5-digit, non-sequential number. Animal ids remained blinded to all data analysts until measurements were completed.

\section{Polyp evaluation}

Following $72 \mathrm{~h}$ of fixation, the fixative was drained from each intestinal tract and the tissue was transferred to $70 \%$ ethanol and maintained at $4{ }^{\circ} \mathrm{C}$ until analysis. For analysis, each intestinal tract was split longitudinally, spread open with mucosal surface exposed for observation and photographed in PBS on a Nikon SMZ1500 dissecting microscope by investigators blinded to the animal treatment condition. Micrographs of the entire mucosal surface were separated into manageable segments and evaluated for the presence of abnormal lesions that were clearly distinguishable from Peyer's Patches. Lesions were marked and measured using NIS elements software (Nikon). Following completion of polyp annotation and measurement, the animal treatment conditions were un-blinded and summary statistics generated comparing total polyp number, total polyp area and polyp size per treatment condition by ANOVA followed by Tukey's HSD post-test.

\section{Gastric gross and histological assessment}

At necropsy, the stomach was removed from each animal and opened along the greater curvature for exposure of the gastric mucosa. The tissue was gently rinsed with PBS, examined grossly and photographed using a dissecting microscope. Then all stomachs were fixed in $10 \%$ neutral buffered formalin for $48-72 \mathrm{~h}$. Multiple sections from each formalin fixed stomach were processed and embedded in paraffin blocks. Four-micron-thick sections of these tissue blocks were stained with hematoxylin and eosin (H\&E) and examined microscopically by a veterinary pathologist without knowledge of animal treatment identification. The severity and extent of histopathological lesions of inflammation and hyperplasia of gastric mucosa were scored with either grade 1 (minimal lesions affecting $1-10 \%$ of tissue), grade 2 (mild lesions affecting 11-20\%), grade 3 (moderate lesions affecting $21-40 \%$ ) or grade 4 (marked lesions affecting $41-100 \%$ of examined tissue). Ulceration (loss of entire mucosal thickness) of the glandular gastric mucosa was recorded as either present or absent. The highest-grade inflammatory lesion (1-4) was plotted for each mouse and group differences were assessed by Student's $\mathrm{t}$ test.

\section{Immunohistochemistry evaluation}

After imaging completion, fixed intestinal tissues were placed in a modified Swiss roll formation, and embedded in paraffin for sectioning. Paraffin sections of the small and large intestine were stained by routine H\&E protocol. Purified mouse anti $\beta$ catenin antibody (\# 610153) was purchased from BD Bioscience (San Jose, CA) and intestine sections were stained as per the protocol validated by the Research Histology Pathology Imaging Core of MDACC. The $\beta$ catenin staining intensity of each 
polyp was scored at a scale of 0 to 3,0 -no staining, $1+=$ weak staining, $2+=$ mild staining, $3+=$ strong staining. $\mathrm{H}$-scores were generated for each mouse using the following formula: $\mathrm{H}=[1 \times(\%$ polyps $1+)+2 \times(\%$ polyps $2+)+3 \times(\%$ polyps $3+)$ ]. Both $\mathrm{H}$-scores and average percent polyps per staining intensity are shown. Histopathology evaluation was conducted without knowing the identity of the specimens with respect to treatment and group assignment. Group comparisons were conducted on the H-score data using ANOVA followed by Tukey's HSD post-test. Graphs and statistical analyses were produced using GraphPad Prism 7.03 (GraphPad Software, Inc.).

\section{Hematologic evaluation}

Blood samples collected at euthanasia were tested for complete blood cell counts (red blood cells, white blood cells, platelets, hemoglobin and hematocrit) with differential counts (neutrophils, eosinophils, segmented cells, monocytes and lymphocytes) and blood chemistry tests (blood urea nitrogen (BUN), creatinine, alkaline phosphatase, alanine transaminase (ALT), aspartate aminotransferase (AST), albumin, globulin, total protein and total bilirubin). Blood counts and chemistries were compared for differences across treatment groups using ANOVA followed by Tukey's HSD post-test.

\section{Urine prostaglandins}

Urinary prostaglandins are a frequently used measure of systemic COX activity, as they are down-stream metabolites of these enzymes [32]. Urine was collected from the euthanasia chamber and puncture of the urinary bladder at necropsy. For mice with insufficient urine collected, samples were pooled by treatment. Following collection, $100 \mu \mathrm{l}$ urine aliquots were immediately frozen on dry ice and maintained at $-80 \mathrm{C}$ until the time of analysis. Urinary prostaglandin profiles were measured using an Agilent 6460 triple quadrupole chromatograph/mass spectrometer as previously described [33]. Briefly, $50 \mu \mathrm{l}$ of urine was spiked with $100 \mathrm{ng}$ tetraor PGEM-d6, 2,3dinor-PGF1 $\alpha$-d9, and 11-dehydrox-TXB2-d4 (internal standards for urinary metabolites of $\mathrm{PGE}_{2}, \mathrm{PGI}_{2}$, and $\mathrm{TXB}_{2}$ ) followed by derivatization with methanoxyamine hydrochloride solution $(25 \mu \mathrm{g})$. Samples were then incubated at $37^{\circ} \mathrm{C}$ for $30 \mathrm{~min}$. The urinary metabolites were applied to Strata-X $(30 \mathrm{mg})$ reverse phase extraction cartridges (Phenomenex, Milford, MA), eluted with 5\% acetonitrile $(\mathrm{ACN})$ in ethyl acetate, dried with a stream of nitrogen followed with reconstitution in $100 \mu \mathrm{l}$ of $50 \%$ methanol water. To fully quantify urinary COX-2 metabolites, these metabolites were separated by reversedphase HPLC (Agilent 1200, Santa Clara, CA) using Phenomenex Kinetex C18 column $(100 \mathrm{~mm} \times 2.1 \mathrm{~mm}$ I.D., $2.6 \mu \mathrm{m})$ with gradient mobile phase of $0.05 \%$ aqueous acetic acid and $0.05 \%$ acetic acid in methanol: ACN (5: 95). The identification and quantification of these urinary metabolites were carried out using Agilent 6460 triple quadruple mass spectrometer by negative multiple reaction, monitoring the transition of PGEM at $m / z 385$ $\rightarrow$ 336, PGIM set at $m / z 370 \rightarrow 232$ and TXBM at $\mathrm{m} / \mathrm{z}$ $370 \rightarrow 155$. Creatinine levels were used to normalize the final outcome of the urinary COX-2 metabolites.

\section{Results}

We tested the relative ability of sulindac and sulindacPC to reduce polyp count and polyp burden in $A p c^{\min /+}$ mice treated for 3 weeks starting at 7 to 8 weeks of age. Two mice randomized to the sulindac-PC arm became moribund very early in the experiment due to gavage accident and were euthanized. Gavage techniques were reoptimized to avoid any further injury. These mice were replaced in the study and their data are not included in the analyses. No other adverse events were observed. Mice treated with either sulindac or sulindac-PC had significantly reduced polyp count (Fig. 1a). Specifically, polyp count was reduced by approximately $58 \%$ with sulindac treatment and 64\% with sulindac-PC treatment (Fig. 1b). Polyp burden, as measured by intestinal polyp area, was significantly reduced compared to non-treated and PC only treated animals (Fig. 1c). Additionally, the size of the remaining polyps tended to be smaller with significantly lower percentages of $1.0-2.0 \mathrm{~mm}$ polyps in sulindac and sulindac-PC treated mice compared to controls (Fig. 1d). Representative intestinal images are shown with and without polyp annotations (Fig. 1e).

\section{Gl safety}

Stomachs were examined histopathologically for the presence of gastric lesions, which were graded as described above and compared across treatment conditions. Histopathologic lesions observed include: acute and subacute ulceration of the glandular mucosa, and acute and subacute inflammation of the gastric glandular mucosa and submucosa. Lesions of inflammation and hyperplasia of epithelial cells of glandular mucosa indicate mucosal injury and/or healing of preexistent mucosal erosions or ulcers caused by sulindac treatment or stress. Inflammation of glandular gastric mucosa was observed in 7/7 mice from sulindac treated group and in 5/ 6 mice from sulindac-PC treated group. The severity of inflammation of gastric mucosa was significantly greater in the sulindac treated group (2.14 average score) in comparison with the sulindac-PC treated group (1.00 average score, Fig. 2a, $p=0.02$ ). Similarly, the incidence and severity of hyperplastic changes of glandular epithelium of gastric mucosa was higher in the sulindac treated group (5/7 mice and 1.57 average score) in comparison with sulindac-PC treated group (3/6 mice and 


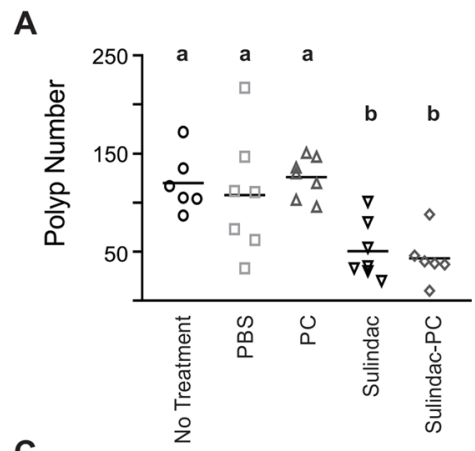

C
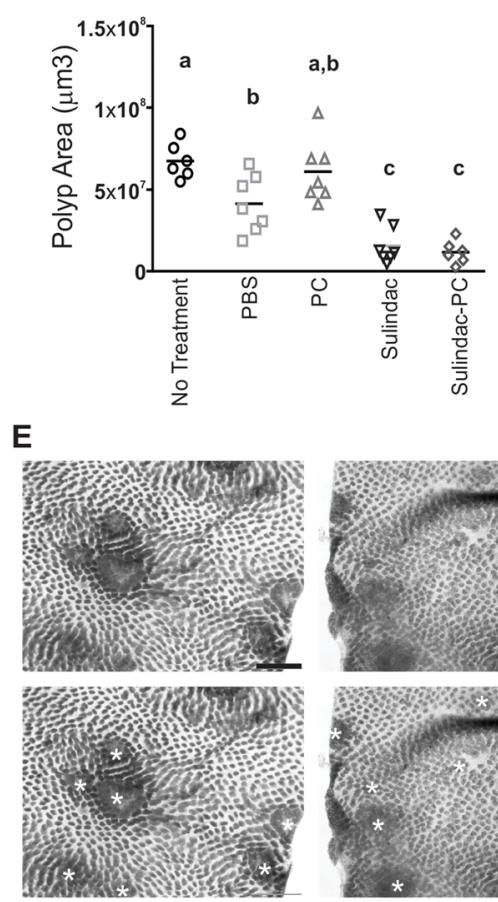

PC

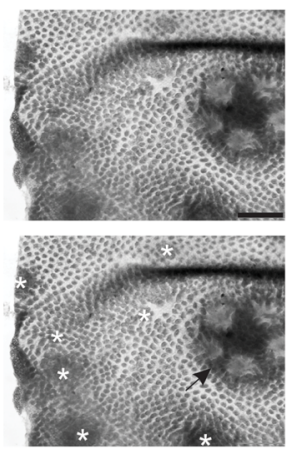

PBS

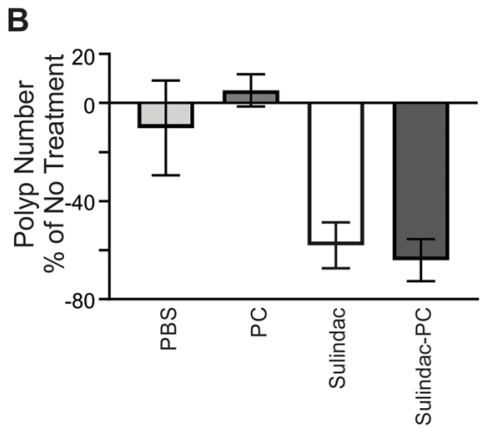

D
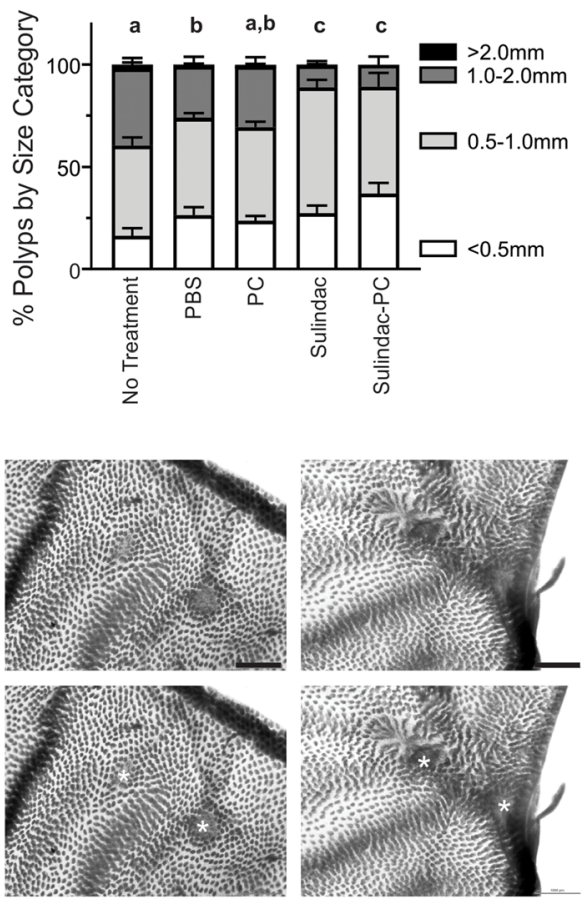

Sulindac

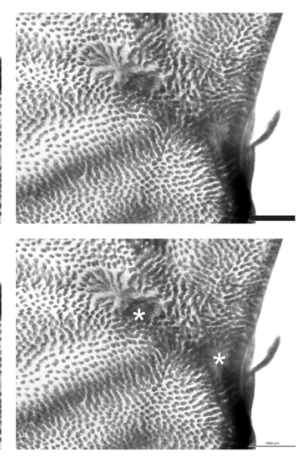

Sulindac-PC

Fig. 1 Sulindac and Sulindac-PC are effective at reducing polyp burden. a Total intestinal polyp number by treatment group No Treatment (O), PBS ( $\square)$, PC $(\Delta)$, Sulindac $(\nabla)$, Sulindac-PC $(\diamond)$, groups with different letters are significantly different from each other. Each point represents data from an individual mouse. $\mathbf{b}$ Percent reduction in polyp count compared to No Treatment group. c Total intestinal polyp area by treatment group $\mathbf{d}$ Percent of polyps are shown by size category and treatment group, groups with different letters have significantly different proportions of 1.0-2.0 mm polyps. Columns = average, bars = standard error of the mean, $N=6-7$ mice per treatment group, each symbol represents one mouse e Representative images of polyp annotations. Tissues are shown without (top) and with (bottom) polyps annotated (*). Arrow indicates a Peyer's patch. Scale bar $=1000 \mu \mathrm{m}$

0.67 average score), though this difference in scores was not statistically significant ( $p=0.13$ ) (Fig. 2b).

Histopathological examination revealed presence of ulceration of gastric mucosa in $2 / 7$ mice treated with sulindac alone, while none of the six mice treated with sulindac-PC had lesions of ulceration, though this difference was not statistically significant (Fig. 2c). Representative photomicrographs of scored lesions are displayed by treatment condition (Fig. 2d), showing normal gastric mucosa in a mouse receiving no treatment (upper left), an acute, focal ulcer in a mouse receiving sulindac alone (upper right, arrow), a focal erosion of the gastric mucosa in a mouse receiving PBS (lower left, arrow), and a micro-erosion of the gastric mucosa with inflammation in a mouse receiving sulindac-PC (lower right, arrow).

\section{Biological activity of sulindac and sulindac-PC}

In addition to polyp reduction, the biological activity of sulindac and sulindac-PC was evaluated by measuring the relative intensity of nuclear $\beta$-catenin staining by immunohistochemistry (IHC), as an indicator of cellular proliferative activity (Fig. 3a-b). Of the polyps remaining in the sulindac and sulindac-PC treatment groups, there 


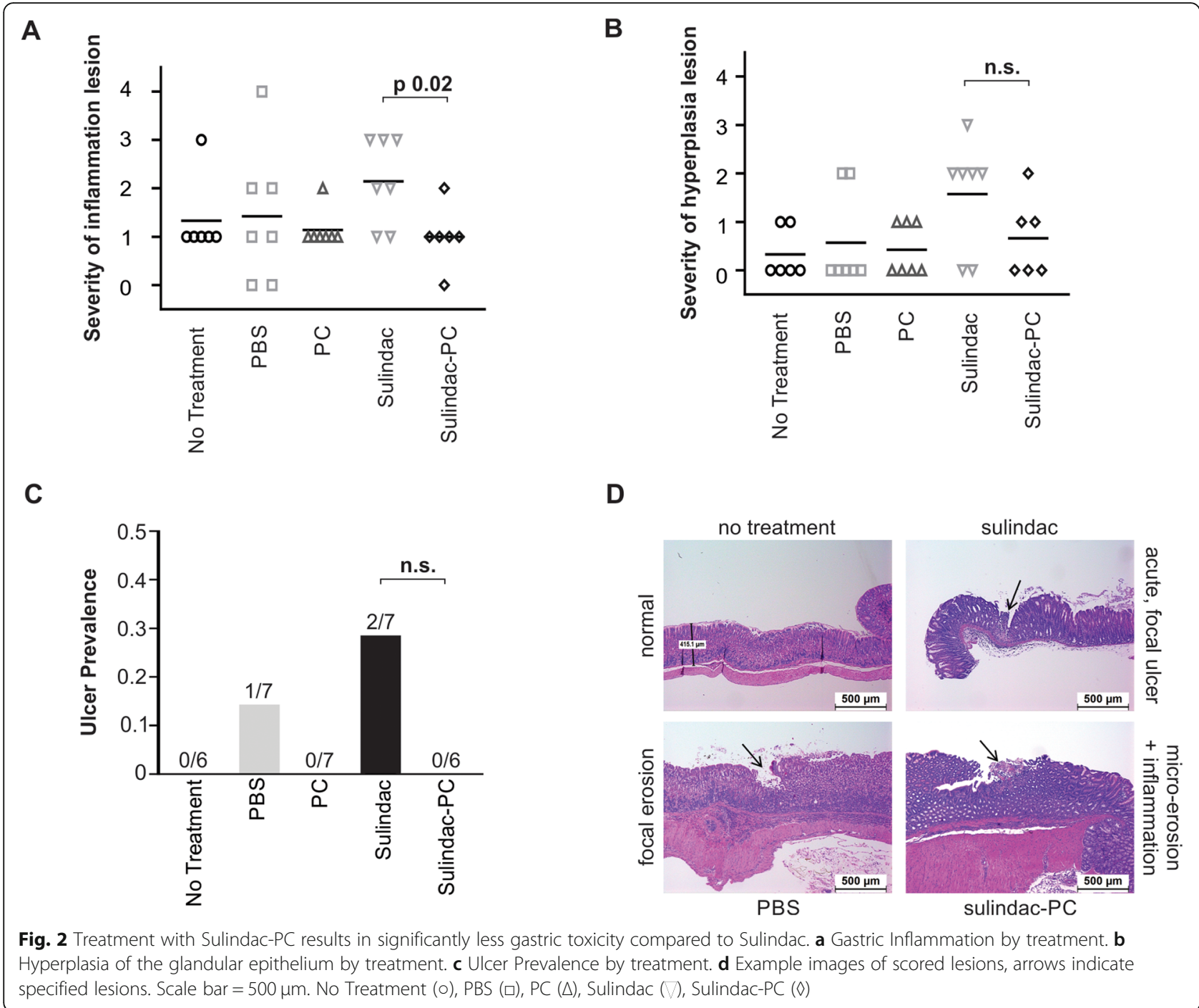

was significantly less nuclear $\beta$-catenin staining compared to controls. Sulindac, like other NSAIDs inhibits the cyclooxygenase (COX) pathway and specifically inhibition of COX-2 may be important for CRC prevention [32]. To assess systemic effects of sulindac and sulindac-PC treatment, we assessed endpoint urinary prostaglandin levels, down-stream metabolites of COX activity, across treatment groups, showing reductions in PGEM and 2,3 dinor-TXB2 in the sulindac and sulindac-PC treated animals with some variability in reductions of additional prostaglandins measured (Fig. 3c).

\section{Animal health}

Treatment did not alter weight gain trajectory of treated mice compared to the non-treated control (data not shown). At the end of each study, blood chemistries (including liver and kidney function tests) and complete blood counts were obtained (Table 2). Differences were noted in the complete blood count between control and sulindac or sulindac-PC treated animals, including increased hematocrit and red blood cell counts and increased mean corpuscle hemoglobin concentration (Table 2).

\section{Discussion}

For effective chemoprevention strategies to be accepted and utilized, the benefits of such treatments must significantly outweigh the risks. Based on substantial concerns over GI toxicity and bleeding, aspirin use for CRC chemoprevention is restricted to relatively small populations. Improving the GI safety of aspirin and non-aspirin NSAIDs is an important step to making these agents safer for chemopreventive use in larger populations. Our findings of decreased stomach toxicity in sulindac-PC mice compared to sulindac alone supports the hypothesis that associating NSAIDs with phospholipids, such as phosphatidylcholine may be an important strategy to 


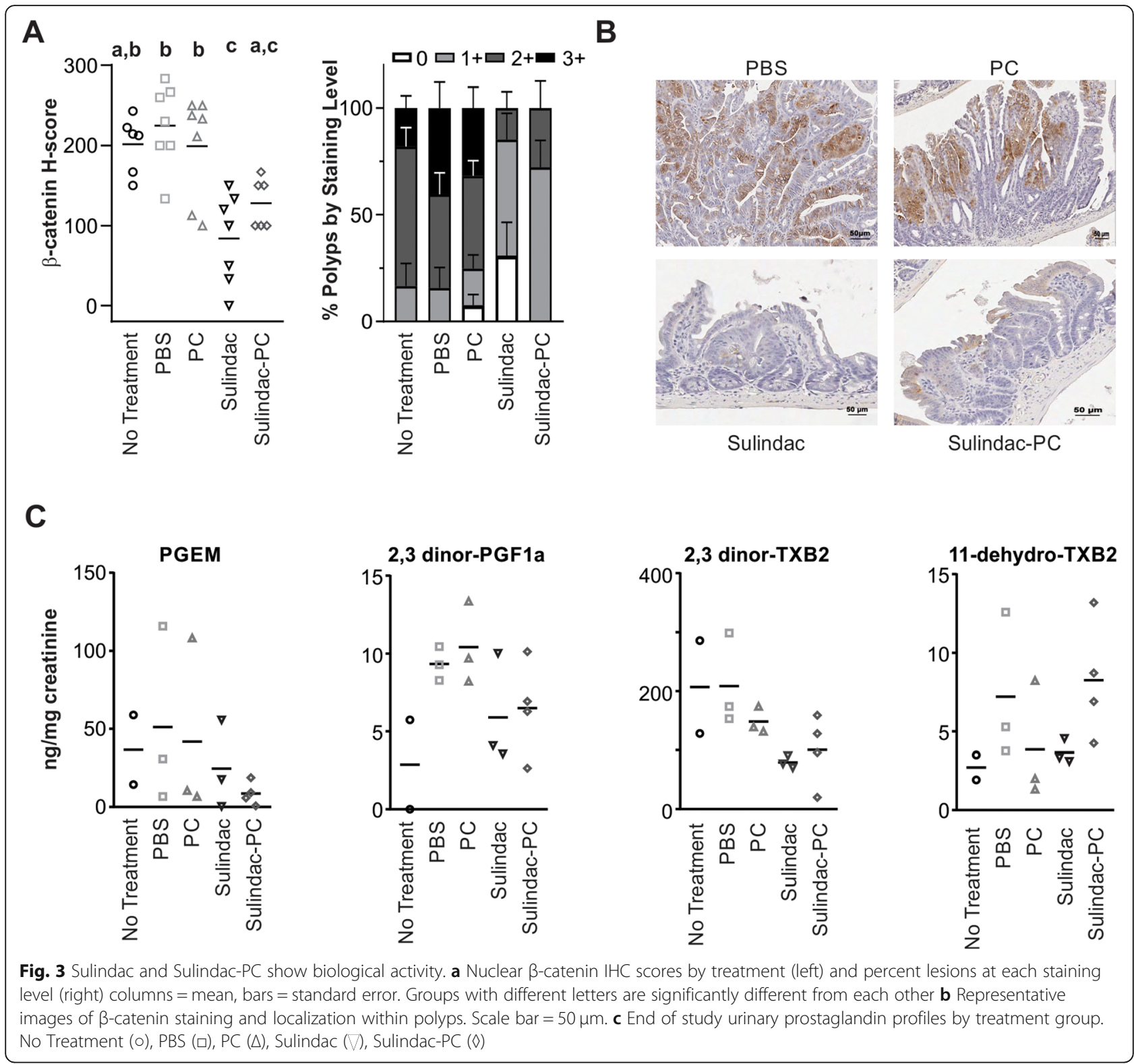

minimize the GI toxicity of prolonged use without compromising chemopreventive efficacy.

These observations clearly demonstrate that sulindacPC treatment resulted in significantly decreased gastric inflammation and may result in decreased hyperplasia of gastric mucosa and ulceration compared with sulindac alone (Fig. 2). The differences in severity of hyperplasia were suggestive of improvements in sulindac-PC, but severity of lesions within treatment groups was heterogenous (Fig. 2b). Further, the very low prevalence of ulcers in the treatment groups did not provide adequate power to detect a significant difference between treatment groups. Several factors likely contributed to these non-significant differences. First, our dose of sulindac $(30 \mathrm{mg} / \mathrm{kg} /$ day $)$ was chosen as the dose needed to reduce polyps with 3 weeks of daily dosing, but is equivalent to approximately half of the daily dose [29] used in a primary chemoprevention trial with FAP patients, who were given $150 \mathrm{mg}$ of sulindac twice daily [34]. Second, the length of treatment in our study was relatively short. Increasing sulindac dose, length of treatment, or both may increase the prevalence of gastric injury observed. Despite these limitations, our results suggest that the addition of PC results in decreased toxicity to the gastric mucosa in comparison to sulindac alone, and therefore supports the important role of PC in protecting gastric mucosa when associated with sulindac treatment. Further, the changes observed in blood counts (Table 2) suggest improvements in the anemia usually associated with polyp burden in the $A p c^{\mathrm{min} /+}$ 
Table 2 Summary hematology results

\begin{tabular}{|c|c|c|c|c|c|c|}
\hline \multicolumn{7}{|c|}{ Blood Chemistry results by treatment group } \\
\hline Blood Chemistry & No Treatment & PBS & PC & Sulindac & Sulindac-PC & $p$ value \\
\hline$n$ & 6 & 7 & 7 & 6 & 5 & \\
\hline Albumin (SD) & $3.5(0.3)$ & $3.4(0.3)$ & $3.3(0.4)$ & $3.7(0.2)$ & $3.5(0.1)$ & 0.3 \\
\hline Alk Phos & $109.8(25.4)$ & $101.9(24.5)$ & $92.6(23.5)$ & $119.0(25.8)$ & $88.2(29.1)$ & 0.3 \\
\hline ALT & $124.7(131.4)$ & $350(352.6)$ & $237.7(267.3)$ & $125.7(42.1)$ & $95.8(62.2)$ & 0.3 \\
\hline AST & $287.8(86.4)$ & $514.3(500.8)$ & $650.1(424.0)$ & $268.7(99.3)$ & $416.5(471.8)$ & 0.3 \\
\hline$n$ & 6 & 7 & 7 & 6 & 4 & \\
\hline BUN & $23.4(4.3)$ & $27.1(4.7)^{*}$ & $18.6(3.4)^{*}$ & $24.1(4.3)$ & $24.3(6.3)$ & 0.03 \\
\hline Globulin & $1.4(0.3)$ & $1.4(0.2)$ & $1.5(0.2)$ & $1.5(0.2)$ & $1.4(0.1)$ & 0.7 \\
\hline Total Protein & $4.9(0.6)$ & $4.9(0.4)$ & $4.8(0.4)$ & $5.2(0.3)$ & $5.0(0.3)$ & 0.3 \\
\hline$n$ & 2 & 1 & 2 & 4 & 4 & \\
\hline Creatinine & $0.24(0.04)$ & 0.20 & $0.24(0.01)$ & $0.24(0.02)$ & $0.22(0.02)$ & 0.5 \\
\hline $\mathrm{n}$ & 0 & 1 & 1 & 0 & 1 & \\
\hline Total Bilirubin & & 0.2 & 0.3 & & 0.2 & NA \\
\hline \multicolumn{7}{|c|}{ Complete Blood Counts by treatment group } \\
\hline Blood Chemistry & No Treatment & PBS & PC & Sulindac & Sulindac-PC & $p$ value \\
\hline$n$ & 6 & 7 & 7 & 6 & 6 & \\
\hline Hemoglobin, g/dL (SD) & $13.5(2.5)$ & $13.4(0.8)$ & $13.2(1.8)$ & $15.7(1.2)$ & $15.3(1.3)$ & 0.03 \\
\hline Hematocrit, \% & $49.6(9.0)$ & $48.1^{\mathrm{a}}(3.3)$ & $47.7^{\mathrm{a}}(5.9)$ & $58.3^{\mathrm{b}}(4.7)$ & $53.2(3.9)$ & 0.01 \\
\hline RBC count, $\times 10 e^{6} / \mu \mathrm{L}$ & $9.6(2.0)$ & $9.3(0.7)^{*}$ & $9.5(1.3)$ & $11.5(0.7)^{*}$ & $10.8(0.8)$ & 0.01 \\
\hline WBC count, $\times 10 \mathrm{e}^{3} / \mu \mathrm{L}$ & $8.7(1.6)$ & $5.7(2.0)$ & $6.9(2.7)$ & $9.4(3.7)$ & $7.5(2.4)$ & 0.11 \\
\hline Platelet count, $\times 10 \mathrm{e}^{3} / \mu \mathrm{L}$ & $881(375)$ & $1049(100)$ & $1056(323)$ & $644(310)$ & 777 (394) & 0.11 \\
\hline
\end{tabular}

Values marked with * are significantly different from each other, but not any other values in that row. Values with different superscript letters are statistically different from each other. For example, columns with ' $a$ ' are significantly different from columns with ' $b$ ', or ' $c$ ', but not different from other columns with ' $a$ '. For example, hematocrit percentages for PBS and PC treated animals are significantly lower than sulindac treated animals, but are not different from each other. $A L T$ Alanine transaminase, AST Aspartate aminotransferase, Alk Phos Alkaline phosphatase, BUN Blood urea nitrogen, $n$ number of animals, RBC Red blood cells, WBC White blood cells

model and is consistent with the polyp reduction observed in animals treated with sulindac or sulindac-PC.

Sulindac has been shown to inhibit $\beta$-catenin expression in the histologically normal appearing colon tissue of patients with the hereditary colorectal cancer syndromes, Hereditary Non-Polyposis Colorectal Cancer, also known as Lynch Syndrome, and FAP [35, 36]. Since the preparation of sulindac with phosphatidylcholine required sonication of the drugs, we confirmed biological activity of these preparations in vivo by demonstrating a reduction of polyp burden, significantly decreased nuclear $\beta$-catenin staining in the remaining polyps and a trend toward decreased urinary prostaglandins of treated mice. While PGEM, 2,3 dinor-TXB2 and 2,3 dinorPGF1a appear to be decreased in sulindac and sulindacPC treated mice, 11-dehydro-TXB2 only appears to be decreased in sulindac treated mice (Fig. 3c). One of the limitations of this analysis is our sample size. While we attempted to collect urine from each animal at the end of the study, we were unable to collect enough volume from many of the mice, resulting in pooled samples and overall reduced numbers. Specifically, we were only able to run prostaglandin levels on two samples for untreated mice, three samples each for PBS, PC and sulindac treated mice, and four samples for sulindac-PC treated mice. The low number of samples and variability of some measures preclude any formal statistical analyses of these data. However, the general decline in urinary prostaglandins of mice treated with either sulindac or sulindac-PC is supportive of systemic COX suppression. Our finding of significantly decreased nuclear $\beta$-catenin in remaining polyps is stronger evidence of the biological activity of sulindac and sulindac-PC and may suggest lower risk for these lesions to recur. Indeed, $\beta$-catenin, COX-2 and P53 staining have been used retrospectively, to show a significant association with adenoma recurrence in a prospective chemoprevention trial [37]. Together, these data support the efficacy, biologic activity and improved GI safety of sulindac combined with a phospholipid, lending critical support to the concept of improved safety for chemopreventive NSAIDs combined with phospholipids. If validated, these findings have the 
potential to significantly expand the portion of the population able to benefit from NSAID based CRC chemoprevention by reducing the risk of GI toxicity. Over time, such increasing use, combined with screening, may lead to profound reductions in CRC incidence.

In sulindac-PC, the sulindac is not covalently associated or crosslinked to the $\mathrm{PC}$, rather the interaction is limited to ionic and hydrogen bonding and is expected to resemble the associations of aspirin-PC and indomethacin-PC as previously published [15]. Although not measured in our study, the association of sulindac and sulindac-PC is not expected to alter the bio-availability or pharmacokinetics/pharmacodynamics of sulindac, as has been demonstrated for aspirin-PC [13].

While our study supports chemopreventive efficacy and improved gastric toxicity of sulindac-PC, the very low incidence of gastric ulceration limits the strength of our conclusions on GI safety. Additionally, our study was conducted over a relatively short duration of 3 weeks. It is possible that treatment over a longer time period may have resulted in additional gastric injury in both control and experimental groups. Now that we have established a method to measure gastric injury in our mice, further studies are needed to determine the consequences of daily oral gavage of sulindac with and without PC for increasing time periods. The dose used in our study was equivalent to approximately $150 \mathrm{mg} /$ day in an adult human [29], whereas a clinical trial in patients with FAP utilized $150 \mathrm{mg}$ twice a day for primary prevention [34]. Increased dosing and extended treatment periods may have improved our ability to detect more substantial differences in gastric toxicity by treatment group.

\section{Conclusions}

Although the $A p c^{\mathrm{min} /+}$ mouse model does not consistently recapitulate the chemopreventive effects of aspirin observed in humans [22-27], our results with sulindacPC provide indirect evidence that the addition of PC improved the GI safety without compromising chemopreventive efficacy. Further, the recently reported aspirin-PC xenograft studies provide more direct evidence of the chemopreventive activity of aspirin-PC [15]. Taken together with prior in vitro, in vivo and clinical trial data, these studies support the consideration of phospholipid or some other polar/zwitterionic lipid, in combination with NSAID preparations to improve the GI safety profile without compromising efficacy.

\section{Abbreviations}

ACN: Acetonitrile; ALT: Alanine transaminase; ANOVA: Analysis of variance; Apc: Adenomatous polyposis coli; ASA: Acetyl salicylic acid; AST: Aspartate aminotransferase; Alk Phos: Alkaline phosphatase; BUN: Blood urea nitrogen COX: Cyclooxygenase; CRC: Colorectal cancer; CVD: Cardiovascular disease; FAP: Familial adenomatous polyposis; FDA: Food and drug administration; Gl: Gastrointestinal; H\&E: Hematoxylin and eosin; HSD: Honestly significant difference; IHC: Immunohistochemistry; min: Multiple intestinal neoplasia; n: Number of animals; NSAID: Non-steroidal anti-inflammatory drug; PBS: Phosphate buffered saline; PC: Phosphatidylcholine; PGEM: Prostaglandin E2 metabolite; PGF1a: Prostaglandin F1alpha; PGI2: Prostaglandin 12; PPI: Proton pump inhibitor; RBC: Red blood cells; SD: Standard deviation; TXB2: Thromboxane B2; WBC: White blood cells

\section{Acknowledgements}

Not Applicable.

\section{Authors' contributions}

JSD contributed to study design, execution, data interpretation and preparation of the manuscript, PKM and PLY contributed to study execution and data interpretation, annotating and measuring intestinal polyps and advising on data presentation. MG contributed to study design, and performed end of study necropsy, gastric toxicity measure and played a significant role in manuscript preparation and data interpretation, DF contributed to study design and execution, providing weekly aliquots of drug preparations and devising methods to combine sulindac and PC. MS contributed to study execution and data interpretation, performing $\mathrm{IHC}$ and generating $\mathrm{H}$-scores. PY contributed to study design and interpretation, specifically advising on urine collection and performing urinary prostaglandin measurements. EH, RD and SK contributed to study design and data interpretation. LML contributed to study design, data interpretation and his lab provided the study drug. DM contributed to study design, execution, and data interpretation, specifically guiding and assisting with the collection and preservation of the intestinal tract and the method for polyp annotation. All authors read and approved the final manuscript.

\section{Funding}

This work was supported by grants from The University of Texas MD Anderson Cancer Center Duncan Family Institute for Cancer Prevention and Risk Assessment, UT MD Anderson Cancer Center Colorectal Cancer Moon Shot, UT MD Anderson Cancer Center Research Histology, Pathology, and Imaging Core (supported by P30 CA16672-39 DHHS/NCI Cancer Center Support Grant), GI SPORE Grant (P50 CA221707) and the National Cancer Institute (2R42CA171408-02A1). None of the funding sources had any role in study design, data collection, analysis or interpretation of findings or drafting of the manuscript.

\section{Availability of data and materials}

Data sharing is not applicable to this article as no datasets were generated or analysed during the current study.

Ethics approval and consent to participate

All procedures were reviewed and approved by MD Anderson's Institutional Animal Care and Use Committee under Dr. David Menter's mouse protocol number 00001187

\section{Consent for publication}

Not Applicable.

\section{Competing interests}

Dr. Lichtenberger is the scientific co-founder of PLx Pharma Inc., and contact PI for the NCI STTR grant, which funded parts of this research (2R42CA171408-02A1). Dr. Sebastian is a member of the medical advisory board of Optrascan. All other authors declare they have no completing interests.

\section{Author details}

'Departments of Epidemiology, The University of Texas, MD Anderson Cancer Center, PO Box 301439, Houston, TX 77230-1439, USA. ²Departments of Gastrointestinal Medical Oncology, University of Texas, MD Anderson Cancer Center, Houston, TX, USA. ${ }^{3}$ Departments of Veterinary Medicine and Surgery, University of Texas, MD Anderson Cancer Center, Houston, TX, USA. ${ }^{4}$ McGovern Medical School, University of Texas Health Science Center, Houston, TX, USA. ${ }^{5}$ Departments of Epigenetics \& Molecular Carcinogenesis, University of Texas, MD Anderson Cancer Center, Houston, TX, USA. ${ }^{6}$ Departments of Palliative, Rehabilitation and Integrative Medicine, University of Texas, MD Anderson Cancer Center, Houston, TX, USA. ${ }^{7}$ Division of Cancer 
Prevention and Population Sciences, University of Texas, MD Anderson Cancer Center, Houston, TX, USA. ${ }^{8}$ Center for Epigenetics \& Disease Prevention, Institute of Biosciences and Technology, Texas A\&M University, Houston, TX, USA.

\section{Received: 3 June 2020 Accepted: 17 August 2020}

Published online: 10 September 2020

\section{References}

1. Chan AT, Giovannucci EL, Meyerhardt JA, Schernhammer ES, Curhan GC, Fuchs CS. Long-term use of aspirin and nonsteroidal anti-inflammatory drugs and risk of colorectal cancer. JAMA. 2005;294:914-23.

2. Chubak J, Whitlock EP, Williams SB, Kamineni A, Burda BU, Buist DS, et al. Aspirin for the prevention of Cancer incidence and mortality: systematic evidence reviews for the U.S. preventive services task force. Ann Intern Med. 2016;164:814-25.

3. Bibbins-Domingo K. Aspirin use for the primary prevention of cardiovascular disease and colorectal cancer: U.S. preventive services task force recommendation statementAspirin use for the Primary Prevention of CVD and CRC. Ann Intern Med. 2016;164:836-45.

4. Hoftiezer JW, Silvoso GR, Burks M, Ivey KJ. Comparison of the effects of regular and enteric-coated aspirin on gastroduodenal mucosa of man. Lancet. 1980;2:609-12.

5. Mo C, Sun G, Lu ML, Zhang L, Wang $Y Z$, Sun $X$, et al. Proton pump inhibitors in prevention of low-dose aspirin-associated upper gastrointestinal injuries. World J Gastroenterol. 2015;21:5382-92.

6. Nagata N, Niikura R, Aoki T, Shimbo T, Kishida Y, Sekine K, et al. Colonic diverticular hemorrhage associated with the use of nonsteroidal antiinflammatory drugs, low-dose aspirin, antiplatelet drugs, and dual therapy. J Gastroenterol Hepatol. 2014:29:1786-93.

7. Stampfer MJ, Jakubowski JA, Deykin D, Schafer Al, Willett WC, Hennekens $\mathrm{CH}$. Effect of alternate-day regular and enteric-coated aspirin on platelet aggregation, bleeding time, and thromboxane A2 levels in bleeding-time blood. Am J Med. 1986;81:400-4.

8. Targownik L. Discontinuing Long-Term PPI Therapy: why, with whom, and how? Am J Gastroenterol. 2018;113:519-28.

9. Hills BA, Butler BD, Lichtenberger LM. Gastric-mucosal barrier - hydrophobic lining to the lumen of the stomach. Am J Physiol. 1983;244:G561-G8.

10. Lichtenberger LM, Graziani LA, Dial EJ, Butler BD, Hills BA. Role of surfaceactive phospholipids in gastric cytoprotection. Science. 1983;219:1327-9.

11. Lichtenberger LM, Wang ZM, Romero JJ, Ulloa C, Perez JC, Giraud MN, et al. Nonsteroidal Antiinflammatory drugs (Nsaids) associate with Zwitterionic phospholipids - insight into the mechanism and reversal of Nsaid-induced gastrointestinal injury. Nat Med. 1995;1:154-8.

12. Lichtenberger LM, Phan T, Okabe S. Aspirin's ability to induce intestinal injury in rats is dependent on bile and can be reversed if pre-associated with phosphatidylcholine. J Physiol Pharmacol. 2011;62:491-6.

13. Angiolillo DJ, Bhatt DL, Lanza F, Cryer B, Dong JF, Jeske W, et al. Pharmacokinetic/pharmacodynamic assessment of a novel, pharmaceutical lipid-aspirin complex: results of a randomized, crossover, bioequivalence study. J Thromb Thrombolysis. 2019;48:554-62.

14. Cryer B, Bhatt DL, Lanza FL, Dong JF, Lichtenberger LM, Marathi UK. Lowdose Aspirin-induced ulceration is attenuated by AspirinPhosphatidylcholine: a randomized clinical trial. Am J Gastroenterol. 2011; 106:272-7.

15. Lichtenberger LM, Phan T, Fang D, Dial EJ. Chemoprevention with phosphatidylcholine non-steroidal anti-inflammatory drugs in vivo and in vitro. Oncol Lett. 2018;15:6688-94.

16. Ritland SR, Gendler SJ. Chemoprevention of intestinal adenomas in the ApcMin mouse by piroxicam: kinetics, strain effects and resistance to chemosuppression. Carcinogenesis. 1999;20:51-8.

17. Wechter WJ, Kantoci D, Murray ED Jr, Quiggle DD, Leipold DD, Gibson KM, et al. R-flurbiprofen chemoprevention and treatment of intestinal adenomas in the APC (min)/+ mouse model: implications for prophylaxis and treatment of colon cancer. Cancer Res. 1997;57:4316-24.

18. Jacoby RF, Seibert K, Cole CE, Kelloff G, Lubet RA. The cyclooxygenase-2 inhibitor celecoxib is a potent preventive and therapeutic agent in the min mouse model of adenomatous polyposis. Cancer Res. 2000;60:5040-4.

19. Murphy EA, Davis JM, McClellan JL, Gordon BT, Carmichael MD. Curcumin's effect on intestinal inflammation and tumorigenesis in the ApcMin/+ mouse. J Interf Cytokine Res. 2011;31:219-26.
20. Paulsen JE, Elvsaas IK, Steffensen IL, Alexander J. A fish oil derived concentrate enriched in eicosapentaenoic and docosahexaenoic acid as ethyl ester suppresses the formation and growth of intestinal polyps in the min mouse. Carcinogenesis. 1997;18:1905-10.

21. Chiu CH, McEntee MF, Whelan J. Sulindac causes rapid regression of preexisting tumors in $\mathrm{min} /+$ mice independent of prostaglandin biosynthesis. Cancer Res. 1997;57:4267-73.

22. Barnes CJ, Lee M. Chemoprevention of spontaneous intestinal adenomas in the adenomatous polyposis coli min mouse model with aspirin. Gastroenterology. 1998;114:873-7.

23. Chiu CH, McEntee MF, Whelan J. Discordant effect of aspirin and indomethacin on intestinal tumor burden in Apc (min/+)mice. Prostaglandins Leukot Essent Fatty Acids. 2000;62:269-75.

24. Mahmoud NN, Dannenberg AJ, Mestre J, Bilinski RT, Churchill MR, Martucci $C$, et al. Aspirin prevents tumors in a murine model of familial adenomatous polyposis. Surgery. 1998;124:225-31.

25. Reuter BK, Zhang XJ, Miller MJS. Therapeutic utility of aspirin in the ApcMin/+ murine model of colon carcinogenesis. BMC Cancer. 2002;2:19.

26. Sansom OJ, Stark LA, Dunlop MG, Clarke AR. Suppression of intestinal and mammary neoplasia by lifetime administration of aspirin in Apc ( $\mathrm{min} /+)$ and Apc (min/+), Msh2(-/-) mice. Cancer Res. 2001;61:7060-4.

27. Wang R, Wang Y, Gao Z, Qu X. The comparative study of acetyl-11-ketobeta-boswellic acid (AKBA) and aspirin in the prevention of intestinal adenomatous polyposis in APC (min/+) mice. Drug Discov Ther. 2014;8:2532.

28. Lynch PM. Chemoprevention of familial adenomatous polyposis. Familial Cancer. 2016;15:467-75.

29. Nair AB, Jacob S. A simple practice guide for dose conversion between animals and human. J Basic Clin Pharm. 2016;7:27-31.

30. Huang Y, Lichtenberger LM, Taylor M, Bottsford-Miller JN, Haemmerle M, Wagner MJ, et al. Antitumor and Antiangiogenic effects of Aspirin-PC in ovarian Cancer. Mol Cancer Ther. 2016;15:2894-904.

31. Sulindac Compound Summary. https://pubchem.ncbi.nlm.nih.gov/ compound/Sulindac. Accessed 8 Apr 2020.

32. Shiff SJ, Rigas B. The role of cyclooxygenase inhibition in the antineoplastic effects of nonsteroidal antiinflammatory drugs (NSAIDs). J Exp Med. 1999; 190:445-50.

33. Pan $Y$, Jiang $Y$, Tan $L$, Ravoori MK, Gagea M, Kundra V, et al. Deletion of cyclooxygenase-2 inhibits K-ras-induced lung carcinogenesis. Oncotarget. 2015;6:38816-26.

34. Giardiello FM, Yang WW, Hylind LM, Krush AJ, Petersen GM, Trimbath JD, et al. Primary chemoprevention of familial adenomatous polyposis with sulindac. N Engl J Med. 2002;346:1054-9.

35. Koornstra JJ, Rijcken FE, Oldenhuis CN, Zwart N, van der Sluis T, Hollema H, et al. Sulindac inhibits beta-catenin expression in normal-appearing colon of hereditary nonpolyposis colorectal cancer and familial adenomatous polyposis patients. Cancer Epidemiol Biomark Prev. 2005;14:1608-12.

36. Boon EM, Keller JJ, Wormhoudt TA, Giardiello FM, Offerhaus GJ, van der Neut $R$, et al. Sulindac targets nuclear beta-catenin accumulation and Wnt signalling in adenomas of patients with familial adenomatous polyposis and in human colorectal cancer cell lines. Br J Cancer. 2004;90:224-9.

37. Brand L, Munding J, Pox CP, Ziebarth W, Reiser M, Huppe D, et al. Sscatenin, cox-2 and p53 immunostaining in colorectal adenomas to predict recurrence after endoscopic polypectomy. Int J Color Dis. 2013;28:1091-8.

\section{Publisher's Note}

Springer Nature remains neutral with regard to jurisdictional claims in published maps and institutional affiliations. 